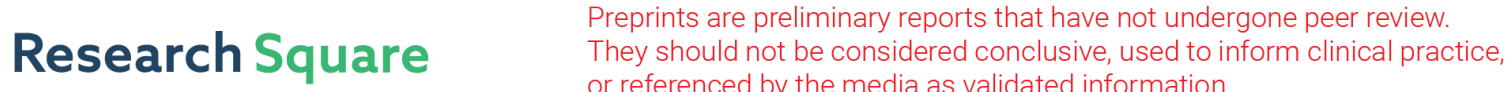 or referenced by the media as validated information. \\ Performance Analysis of ACO-OFDM NOMA for VLC Communication
}

\author{
Jayashree Pradhan \\ National Institute of Technology Rourkela \\ Pratiksha Holey \\ National Institute of Technology Rourkela \\ Vinod Kiran Kappala \\ National Institute of Technology Rourkela \\ Santos Kumar Das ( $\nabla$ dassk@nitrkl.ac.in ) \\ National Institute of Technology Rourkela
}

\section{Research Article}

Keywords: Visible light communication, NOMA, Power allocation, SIC, ACO-OFDM.

Posted Date: December 29th, 2021

DOI: https://doi.org/10.21203/rs.3.rs-1006678/v1

License: (9) This work is licensed under a Creative Commons Attribution 4.0 International License.

Read Full License 


\title{
Performance Analysis of ACO-OFDM NOMA for VLC Communication
}

\author{
Jayashree Pradhan, Pratiksha Holey, Vinod Kiran Kappala \\ and Santos Kumar Das
}

Department of Electronics and Communication, National Institute of Technology, Rourkela, Rourkela, 769008, Odisha, India.

*Corresponding author(s). E-mail(s): dassk@nitrkl.ac.in;
Contributing authors: jayashreepradhan12@gmail.com;
pratikshaholey95@gmail.com; vinodkiran427@gmail.com;

\begin{abstract}
Visible light communication (VLC) is seeking a lot of attention in the recent years due to high bandwidth, low cost, ease of implementation. VLC can be used for illumination as well as communication at the same time. Light emitting diode (LED) acts as a transmitter for data transmission and photo detector is used at the receiver side. Intensity Modulation (IM) is used to convert electrical signal into optical signal where only real and positive signal need to be transmitted. Optical orthogonal frequency division multiplexing (O-OFDM) is used in the VLC to enhance the bandwidth limitation due to LED. Using OOFDM for VLC does not provide the massive connectivity in an multi-user environment. A Non orthogonal multiple access (NOMA) is the further expansion where user can use both the time and frequency resources but distinguished in power domain with successive interference cancellation (SIC) at the receiver to decode the signal of each user. Also, Asymmetrically clipped optical orthogonal frequency division multiplexing (ACO-OFDM) is used to get positive signal with enhanced spectral efficiency. The proposed method is evaluated analytically and using simulation in terms of bit error rate (BER).
\end{abstract}

Keywords: Visible light communication, NOMA, Power allocation, SIC, ACO-OFDM. 


\section{Introduction}

Visible light communication (VLC) is a promising technology for next generation high-speed wireless communication in an indoor scenario. VLC operates in the unlicensed spectrum with high security, large bandwidth and provides strong immune to electromagnetic interference. These advantages drive VLC for broad area of application such as military application, hospital area, flight during travel and underwater communication Chen et al (2017). VLC has been developed to support the needs of fifth generation $(5 \mathrm{G})$ wireless communication and Internet-of-Things (IoT) terminals for indoor communication with higher data rate Mohsan and Amjad (2021). Most of the research in VLC communication is devoted to establish communication under line of sight (LOS) gain of the channel Rodoplu et al (2020).

Apart from the advantages, VLC is limited in operation due to the bandwidth constraint of LED (i.e operates in $\mathrm{MHz}$ ), due to the internal $\mathrm{RC}$ circuit Yeh et al (2015). There are different approaches proposed in the literature to enhance the bandwidth, one such technique is optical orthogonal frequency division multiplexing (OOFDM) Lee et al (2012). Further OOFDM is categorized into three types i.e. Asymmetrically clipped optical orthogonal frequency division multiplexing (ACO-OFDM), DC-biased optical orthogonal frequency division multiplexing (DCO-OFDM) and Asymmetrically clipped DC-biased optical orthogonal frequency division multiplexing (ADO-OFDM). Out of them ACO-OFDM is power efficient at lower SNR Saju et al (2015). DCOOFDM has extra bias circuit though it has high spectral efficiency at higher SNR. Asymmetrically clipped DC-biased optical (ADO-OFDM) is proposed for higher spectral efficiency, which increases the receiver design complexity and also effect of clipping noise Hameed et al (2021), Dissanayake and Armstrong (2013).

The further improvement can be obtained using different modulation technique of higher order QAM. Different multiple access (MA) techniques are proposed for multiuser environment operating at different frequency (Orthogonal frequency division multiple access), time (Time division multiple access), and space (space division multiple access) Lian and Brandt-Pearce (2019). OFDMA is one such MA technique that operates in different frequencies for each user, such that each frequency is orthogonal in nature and using OFDMA degrades the spectral efficiency Al-Ahmadi et al (2018). To overcome the above constraint Non-orthogonal multiple access (NOMA) is used to operate in the same frequency band and time block with different power allocation among the users. NOMA can be implemented efficiently for the VLC communication due to high spectral efficiency and multi-user communication Benjebbour et al (2013). OFDM is limited in operation due to Peak to average power ratio (PAPR) problem that cab be reduced by using NOMA. Single carrier frequency division multiplexing (SCFDM) is performed on NOMA to reduce the PAPR but the method achieved low data rate Ye et al (2017). 
Few of the research work using OFDMA-NOMA with optimum power allocation ratios (PAR) for uplink and downlink is analyzed for multiuser with specific frequency Lin et al (2017); Lian and Brandt-Pearce (2020). QAM OFDM is used in the VLC to improve the bandwidth of LED and is suitable for NOMA application Komine and Nakagawa (2004). In Verma and Selwal (2018); Selvendran et al (2019), a 4 QAM OFDM VLC is studied in optisystem using laser and white LED as transmitter source respectively. In Wang et al (2017), Marshoud et al (2017) the authors approximated the closed-form expression under noisy CSI is obtained for different dimming control techniques of NOMA-VLC model which results in lower data rate.

In Marshoud et al (2015), the transmitter side with superposition coding (SC) is used and successive interference cancellation (SIC) is used at the receiver side. A gain ratio power allocation (GRPA) technique to maximize the sum rate for users is introduced for higher order of modulation. In Fu et al (2018), the authors explains the enhanced power allocation algorithm (EPA) for multicarrier, which allocates power at both user level and subcarrier level. An OFDM NOMA VLC, is designed for LOS but the constraint on type of optical OFDM is not explained Ren et al (2018).

DCO-OFDM NOMA is further studied in Shi et al (2019) where DCOOFDM based on Zero-tailed approach is proposed with subcarrier power allocation and dynamic power allocation for OFDM-NOMA based VLC systems. For low SNR the average optical power of ACO-OFDM is better than DCO-OFDM and also extra bias circuit is not needed in case of ACO-OFDM. . In Liu et al (2019), Barrami et al (2014) a co-operative NOMA-based DCOOFDM VLC system is analysed with DCO OFDM. In Deepthi and Visalakshi (2021) proposed optical OFDM method for high data rate including MIMO OFDM, but this method is limited for NLOS VLC. Based on the above limitations, this work proposes an ACO-OFDM NOMA for LOS and Non-LOS channel condition in a multi-user environment.

The main contributions of papers are as follows:

- An OFDM-NOMA VLC communication system is designed for both LOS and NLOS environment.

- OFDM-NOMA VLC is implemented for a 2-user indoor scenario with optimum power allocation.

- A closed form expression of the proposed system is derived and performance is evaluated in terms of BER.

The remaining part of the paper is organized as follows. Section II describes the system model of VLC communication. Section III presents the proposed ACO-OFDM for NOMA-VLC. Section IV presents the simulations and results of the proposed method. Section $\mathrm{V}$, finally provides the concluding remarks. 


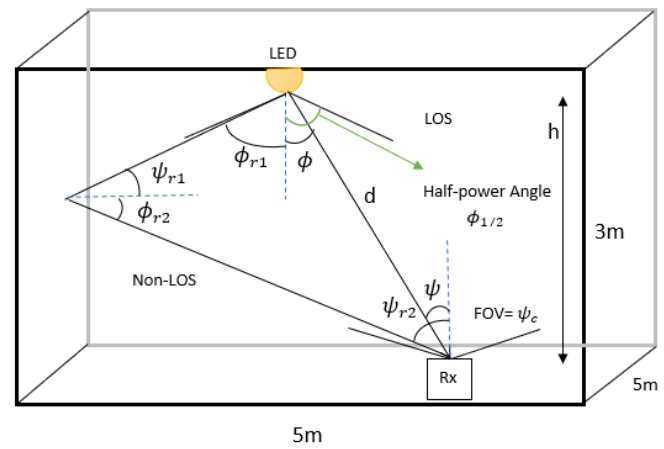

Fig. 1 VLC channel model

\section{Channel and System Model}

A single LED is used to transmit the data in an indoor environment, where it supports for multi-user by modulation the intensity of the light in both LOS and NLOS scenario. The power is divided for two user, where the user are located at different distance. To calculate the optimal response of power for both LOS and NLOS using bit error rate among the users is the backbone of system.

\subsection{The VLC Channel}

VLC channel gain for LOS scenario where optical signals received directly in an indoor communication, can be represented as Jiang et al (2020)

$$
H_{L O S}=\frac{\left(m_{l}+1\right) A_{z}}{2 \pi d^{2}} \cos ^{m_{l}}(\phi) g(\psi) T_{s}(\psi) \cos (\psi),
$$

where $\phi$ is the angle of irradiance with the axis normal to the surface of transmitter, $\psi$ is an incidence angle with the axis normal to the receiver surface, $T_{s}(\psi)$ is the transmission coefficient for optical filter, $\mathrm{g}(\psi)$ is the concentrator gain and $d$ is the distance between the transmitter and the receiver. $A_{z}$ is the area of photo detector. The order of Lambertian emission denoted as $m_{l}$, which is expressed as

$$
m_{l}=\frac{\ln (2)}{\ln \cos \left(\phi_{1 / 2}\right)},
$$

where $\phi_{1 / 2}$ is the semi-angle at half illuminance of an LED. To reduce optical noise due to ambient light, an optical filter is integrated such that the signalto-noise ratio (SNR) is improved. Optical concentrator at a receiver whose gain can be derived as,

$$
g(\psi)=\frac{n^{2}}{\sin ^{2}\left(\psi_{c}\right)} \text { for } 0 \leq \psi \leq \psi_{c},
$$


where $\psi_{c}$ denotes the field of view (FOV) at the receiver and refractive index $n$. In Non-LOS environment the reflections from wall are taken into account and hence the corresponding VLC channel for Non-LOS indoor can be expressed as Komine and Nakagawa (2004),

$$
\begin{array}{r}
H_{N o n-L O S}=\frac{\left(m_{l}+1\right) A_{z}}{2\left(\pi d_{1} d_{2}\right)^{2}} d A_{\text {wall }} \cos ^{m_{l}}\left(\phi_{r 1}\right) \cos \left(\psi_{r 1}\right) \\
\cos \left(\phi_{r 2}\right) \cos \left(\psi_{r 2}\right) T_{s}\left(\psi_{r 2}\right) g\left(\psi_{r 2}\right),
\end{array}
$$

where $d A_{\text {wall }}$ is the effective area of the wall, $d_{1}$ and $d_{2}$ denotes the distances from LED transmitter to wall and from wall to photo detector respectively. $\phi_{r 1}$ and $\phi_{r 2}$ are the corresponding angle of irradiance from LED and wall respectively. $\psi_{r 1}$ and $\psi_{r 2}$ are the angle of incidence to wall and photo detector respectively as shown in FIg. 1 Ghassemlooy et al (2019). The overall channel gain is the addition of both LOS and NLOS gain components derived as,

$$
H=H_{L O S}+H_{N L O S}
$$

\subsection{NOMA VLC Transmission}

In NOMA, the LED transmits real and positive signal to the users placed at variable distances, which associates with different power accordingly. Consider a signal is transmitted with power $P_{i}$ for $i^{\text {th }}$ user out of total $N$ users. Hence the total transmitted signal is derived as,

$$
x=\sum_{i=1}^{N} \sqrt{P_{i}} x_{i},
$$

where $x_{i}$ is superimposed signal of the $i^{t h}$ user and $i=1, \cdots, N$. For simplicity, assume that $N=2$ i.e. 2 users, $i=1$ denotes the far user hence allocated with high power whereas $i=2$ is the near user from receiver and allocated with less power i.e. $P_{2}=\left(1-P_{1}\right)$. The received signal in time domain written in Marshoud et al (2017).

$$
y=h \bigotimes \sum_{i=1}^{N} \sqrt{P_{i}} x_{i}+w,
$$

where $h$ is the channel coefficient from transmitter to the $i^{t h}$ user in time domain, $w$ is used to denote the additive white Gaussian noise (AWGN) with zero mean and $\sigma_{n 2}$ variance. 


\section{Proposed ACO-OFDM NOMA for VLC Communication}

Fig. 2 represents the block diagram representation of ACO-OFDM NOMA system. Consider a 2-user scenario where, data from user each user are QAM modulated and then allocated with power $P_{1}$ and $P_{2}$ respectively. In ACOOFDM only odd subcarrier are used for data and the pilots, modulated data are fed to Hermitian symmetry block, which produces the real data. IFFT converts data from frequency domain to time domain signal. A cyclic prefix is added to eradicate the effect of ISI and zero clipping are performed to get the real and positive signal which are directly fed to transmitter. At the receiver pilots are removed to get original signal after removing cyclic prefix and performing FFT. As intensity modulation (IM) as well as direct detection

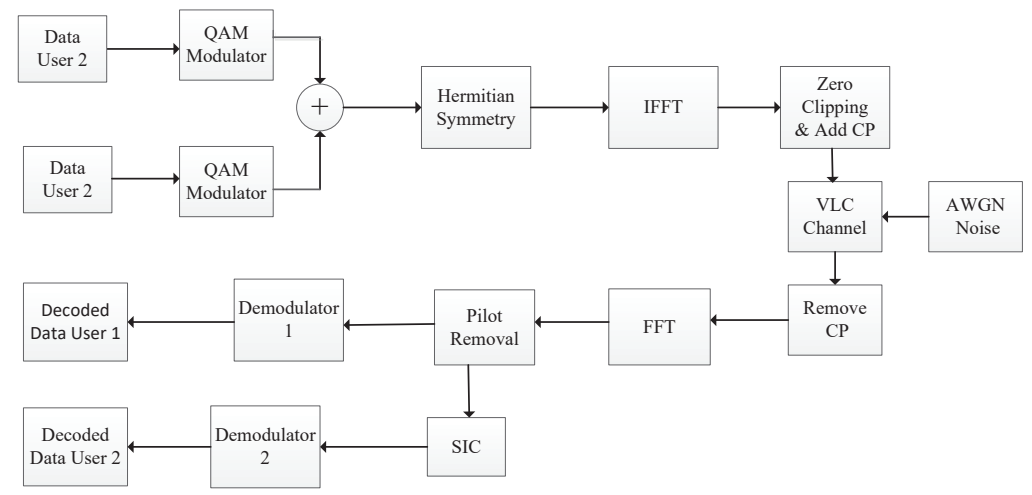

Fig. 2 Block diagram representation of ACO-OFDM for NOMA system

(DD) techniques are used in VLC to send the information for which phased components cannot be used to convey the message. Light intensity of the LED is modulated with the help of transmitted electrical signal which must be real and positive in nature. In DCO and ACO-OFDM techniques the Hermitian symmetry is applied to get the real data and then negative signal are clipped to zero to get positive signal. In proposed work basic model for ACO-OFDM is used for NOMA-VLC system. The SIC scheme is applied after equalization to get modulated decoded data for the another user. Demodulation is performed to retrieve the actual data. The corresponding received signal in frequency domain can be derived from equation (7) as,

$$
Y=H \times \sum_{i=1}^{N} \sqrt{P_{i}} X_{i}+W
$$


where $H$ is the channel impulse response. The received signal divided by a factor of $H \sqrt{P_{1}}$ to user 1 is expressed as,

$$
Y_{1}=X_{1}+\sum_{i=2}^{N} \sqrt{\frac{P_{i}}{P_{1}}} X_{i}+\frac{W}{H \sqrt{P_{1}}},
$$

After removing the term of $X 1$ from above equation and dividing the received signal by $H \sqrt{P_{2}}$, the decoded signal for user 2 as follows,

$$
Y_{2}=X_{2}+\sum_{i=3}^{N} \sqrt{\frac{P_{i}}{P_{2}}} X_{i}+\frac{W}{H \sqrt{P_{2}}},
$$

Similarly, all the $N$ signals by SIC can be decoded. From the received signal, the SNR is calculated to analyze the BER of the signal at receiver. Consider a 4 QAM constellation diagram where points on constellation are represented with $\left( \pm \beta_{1} \pm \beta_{2}\right)$ and $j\left( \pm \beta_{1} \pm \beta_{2}\right)$. The transmitted signal in terms of constellation points is represented as,

$$
x=\sqrt{P_{1} P_{t}}\left(A_{I 1}+j A_{Q 1}\right)+\sqrt{P_{2} P_{t}}\left(A_{I 2}+j A_{Q 2}\right),
$$

where $P_{t}$ is the total transmitted power, $A_{I i}$ and $A_{Q i}$ represents real and imaginary part for $i^{\text {th }}$ user. Probability of error is calculated by calculating probability of error for each bit as

$$
P e_{b 1 i}=\sum_{j, k=0}^{3}\left(P_{e_{b 1 i}} \mid s_{1}=j, s_{2}=k\right)
$$

where $s_{1}$ and $s_{2}$ are the signal for user 1 and 2. Considering the case, now using equation (12) the probability of error for this point on constellation is given as,

$$
P e_{b 11}=\mathbb{Q}\left(\frac{\left(\sqrt{P_{1}}-\sqrt{P_{2}}\right) H}{\sigma}\right)
$$

Similarly, calculating probability of error for each point and adding to get the final expression for probability of error for user 1 and for user 2 considering the interference of user 1 which are given as follows, equation (14) and equation (15) for user 1 and 2 respectively.

$$
\begin{aligned}
B E R_{1}= & \frac{1}{2} \mathbb{Q}\left(\frac{H}{2 \sigma}\left(\sqrt{P_{1}}+\sqrt{P_{2}}\right)\right)+ \\
& \frac{1}{2} \mathbb{Q}\left(\frac{H}{2 \sigma}\left(\sqrt{P_{1}}-\sqrt{P_{2}}\right)\right) .
\end{aligned}
$$




$$
\begin{aligned}
B E R_{2}= & \mathbb{Q}\left(0.54 \times \sqrt{\frac{P_{2} H}{P_{1} 2 \sigma}}\right) \\
& -2 \mathbb{Q}\left(4 \times\left(\sqrt{\frac{H}{2 \sigma}}+\sqrt{\frac{P_{2} H}{P_{1} 2 \sigma}}\right)\right) \\
& +\mathbb{Q}\left(\sqrt{\frac{H}{2 \sigma}}+\sqrt{\frac{P_{2} H}{P_{1} 2 \sigma}}\right) .
\end{aligned}
$$

$\mathbb{Q}(x)$ is the $\mathbb{Q}$ function of $x$ and $\sigma^{2}$ is the noise variance.

\section{Simulation Results}

An indoor environment for ACO-OFDM-NOMA in VLC is designed, which has a dimension of $5 m \times 5 m \times 3 m$. The parameters for simulation of above VLC model is mentioned in Table 1. Here a single LED is used to transmit the data for two user placed at distance of $0.5 \mathrm{~m}$ and $3 \mathrm{~m}$. For LOS communication the field of view (FOV) of photo detector is $85^{\circ}$ where as for Non-LOS the FOV is $70^{\circ}$. The NLOS communication of VLC is having more sensitive towards distance and FOV of receiver. All the reflection from walls and floor are considered for channel designing of NLOS VLC.

Table 1 VLC channel parameters used in simulation

\begin{tabular}{|c|c|c|}
\hline SR.NO. & $\boldsymbol{P A R A M E T E R \boldsymbol { S }}$ & $\boldsymbol{V A L U E S}$ \\
\hline 1 & Power of LED & 3 watt \\
\hline \hline 2 & Field of view (FOV) & 85 degrees \\
\hline \hline 3 & Semi angle at half power & 70 degrees \\
\hline \hline 4 & Refractive index & 1.5 \\
\hline \hline 5 & Photo detector area & $1 \mathrm{e}-4(\mathrm{sq} . \mathrm{m})$ \\
\hline 6 & Room dimensions & $5 \times 5 \times 3(\mathrm{~m})$ \\
\hline 7 & Room Transceiver distance & $3(\mathrm{~m})$ \\
\hline
\end{tabular}

In NLOS case reflections from walls are considered hence the channel gain for each reflection is considered separately and then overall channel gain is used by adding the individual gains. Fig. 3 shows the performance of ACO-OFDM NOMA for VLC in terms of BER It can be observed a higher performance at lower SNR (i.e $20 \mathrm{~dB}$ ) that the BER has improved significantly. Fig. 4 shows the performance of ACO-OFDM NOMA for NLOS. The NLOS performance is evaluated which consists of all the reflections of the room. The BER performance asymptotic with the simulation and BER is high of user 2 with the user 1 as shown in Fig. 4 . 


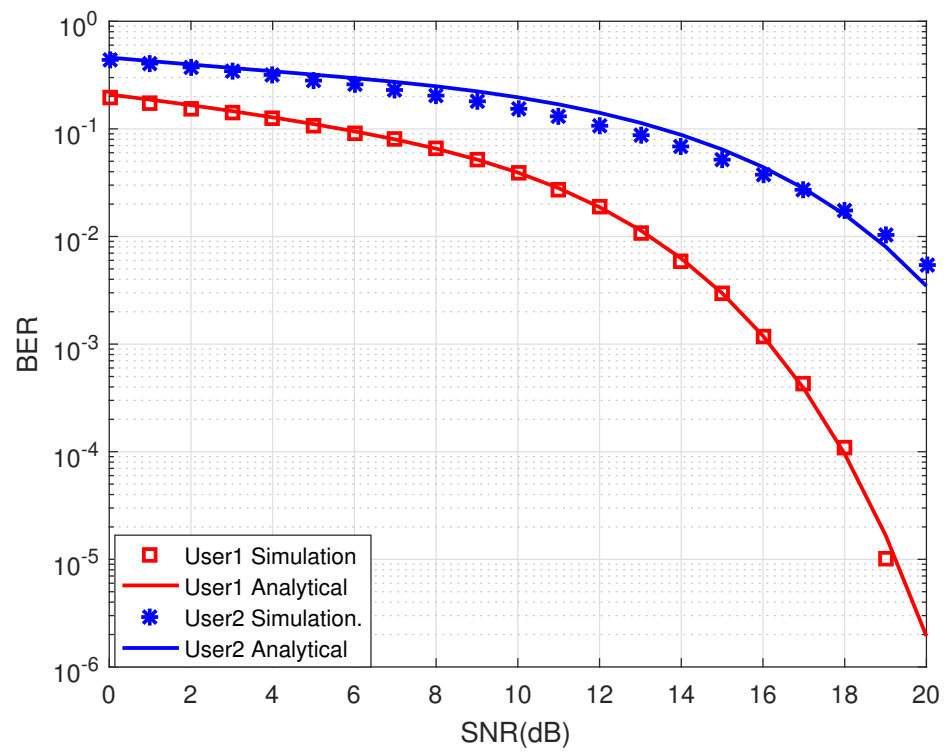

Fig. 3 BER of ACO-OFDM NOMA VLC for LOS

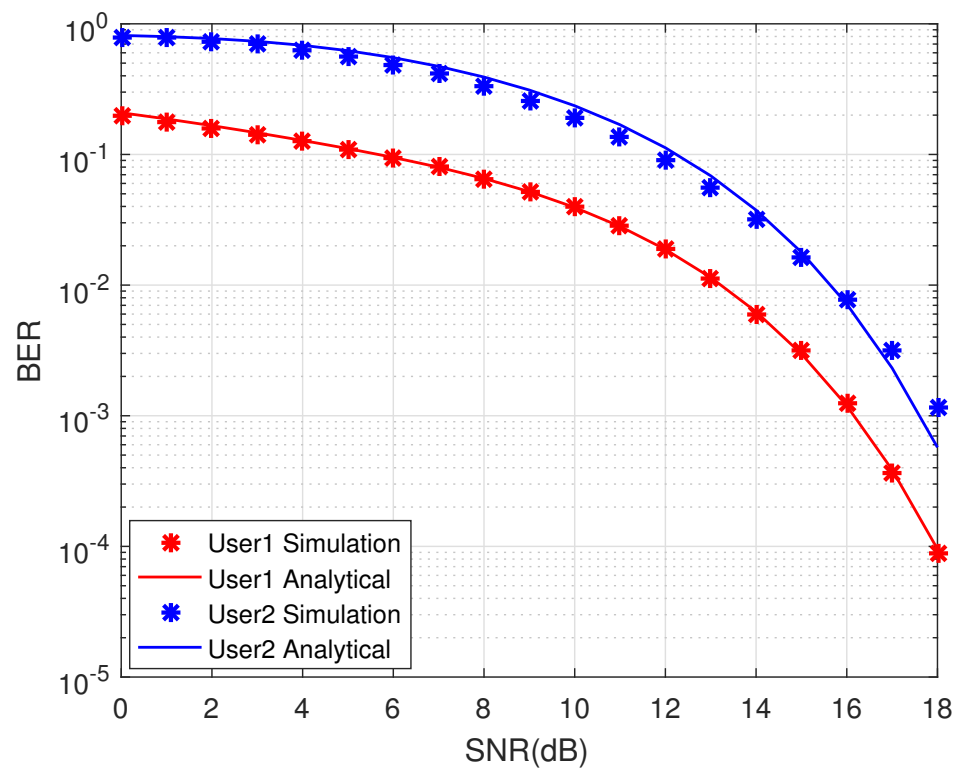

Fig. 4 BER of ACO-OFDM NOMA VLC for NLOS 


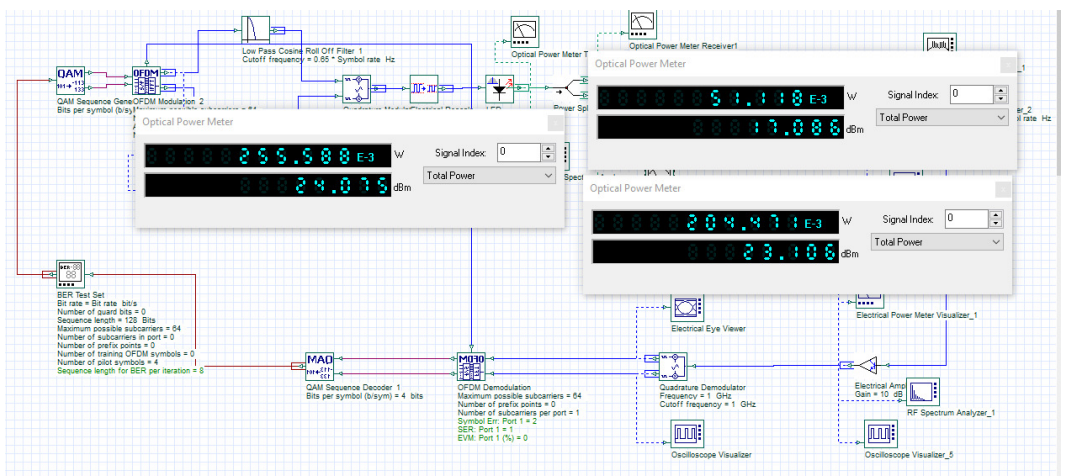

Fig. 5 Optisystem model for VLC OFDM-NOMA

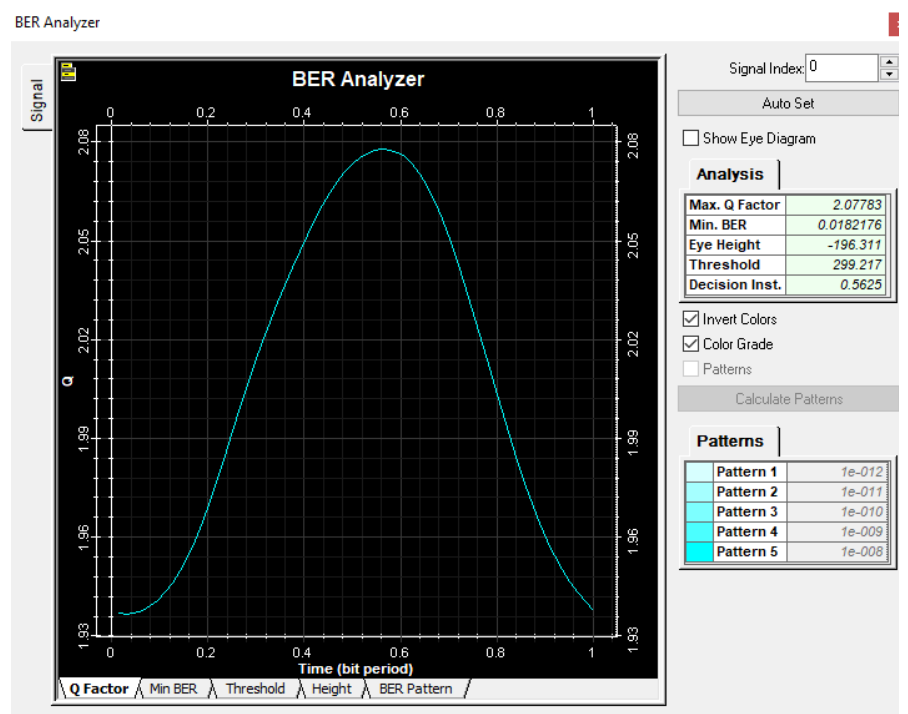

Fig. 6 BER of ACO-OFDM NOMA VLC

ACO OFDM NOMA VLC model is verified experimentally using OptiSystem. Mathematical channel model for LOS and Non-LOS that consists of all the parameter and reflections and the model is designed in MATLAB and cosimulated with Optisystem. An Optisystem simulation of OFDM-NOMA LOS propagation model is as shown in Fig. 5. In this model the pseudo random bit sequence generator is used to generate the continuous no of bits and then NRZ pulse generator is used to supply the data signal for the white LED. The LED emits light which has splitted for 2 users by a different factor i.e $0.2,0.8$ for two user having different distance. The power can be allocated to the 2 users but total power must be same as input power. In Fig. 5 shows the sum 


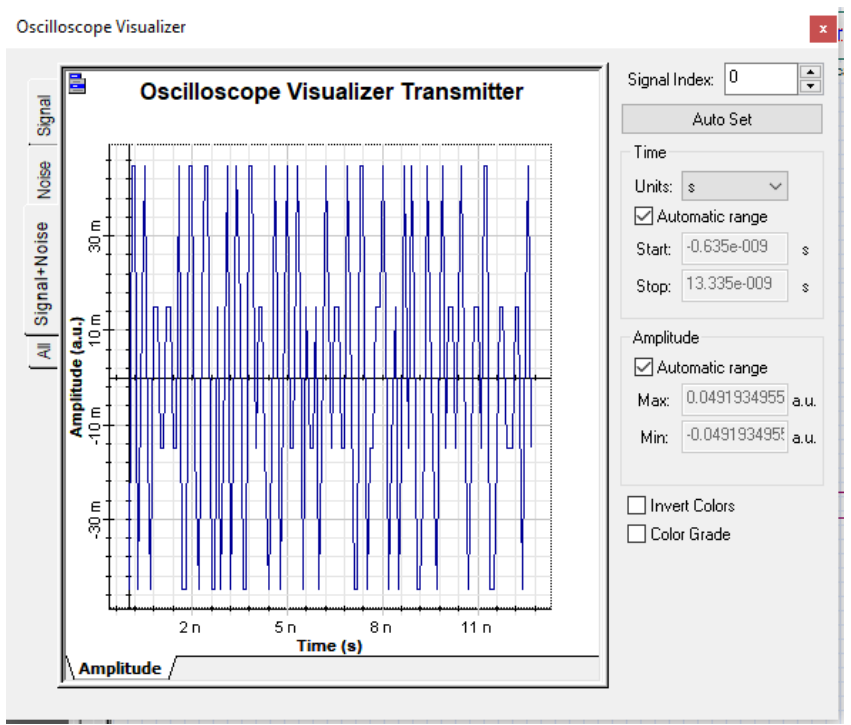

Fig. 7 OFDM-NOMA input data stream vizualizer

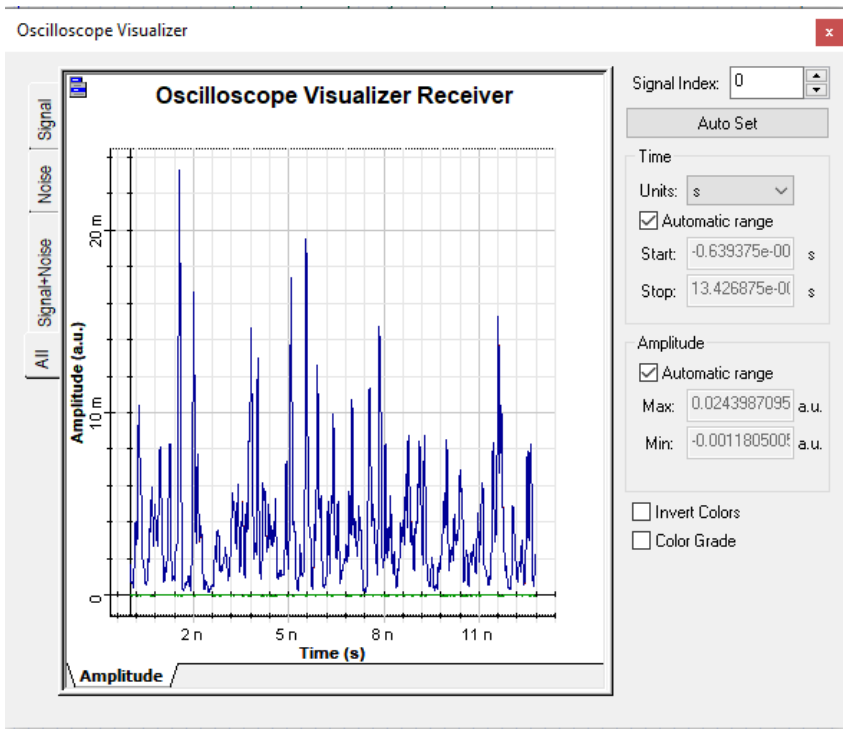

Fig. 8 OFDM-NOMA output data stream vizualizer

of divided power is equal to the total transmitted power, that can be visualized in Fig. 5 of optical power meter reading. The transmitted power is 255.5 $m w$ and that power is divided for two user according to their distance from the source. User 1 is achieved $204.5 \mathrm{mw}$ power and user 2 is achieved 51.1 $m w$ power for the distance $3 m$ and $0.5 m$ respectively. LED encodes the optical information, which is passed through a MATLAB. At the receiving end, 


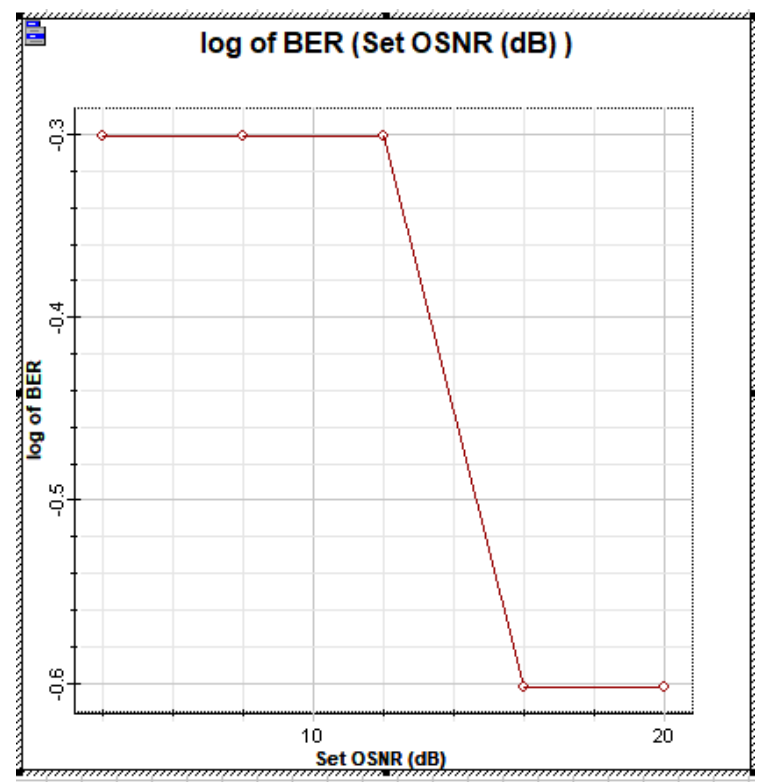

Fig. 9 BER of OFDM-NOMA VLC from optisystem

use a PIN photodiode as a receiver, where the optical signal is converted to electrical signal. Different parameters and their values can be visualized from the layout picture. The BER analyzer output is shows in the Fig. 7, which explains the values of $\min$ BER, max $Q$ factor and eye height, are as shown. The output of BER analyzer explains the error rate of each received samples Selvendran et al (2019). Oscilloscope visualizer is used for viewing data at input and output. The Fig. 7 and Fig. 8 represents the oscilloscope output at transmitter and receiver respectively. The oscilloscope visualizer transmitter helps to visualize the input sampled signal, where oscilloscope visualizer receiver helps to know the output sampled signal after the photodetector. The input sampled signals are bipolar in nature but the received sampled signals are uni-polar in nature can be easily observed from both the oscilloscope visualizer respectively. The optical signal noise ratio (OSNR) component is used to add a defined noise floor level to an optical signal, which is defines as the ratio of total signal power within the signal bandwidth and noise power measured within the window. Fig. 10 represents the result of BER vrs OSNR from the simulation output, which express as the OSNR increases BER reduces, which validates the accuracy of output. The BER rate is high as compared to wireless communication but it is considerable in VLC for high data rate communication Barrami et al (2014). VLC communication is more sensitive towards the distance of transceiver and that can be explained in the Fig. 10. As the distance increases the rate of BER increases is observed. Fig. 8 and Fig. 10 are the output plot from the optisystem, shows the BER response for OSNR and distance respectively. 


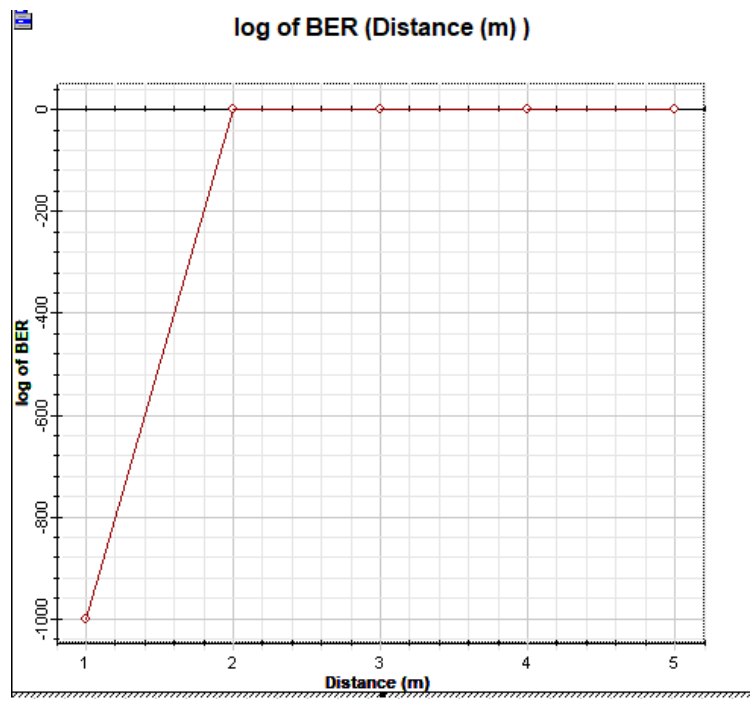

Fig. 10 Performance of BER for OFDM-NOMA VLC

\section{CONCLUSIONS}

The power distribution in VLC model for LOS and Non-LOS indoor communication shows variation in the simulation results with the different FOV of photo detector. The BER performance analysis for ACO-OFDM NOMA for VLC system is analyzed in both analytically and in simulation also. The decoding order is based on the power allocated to each user that is the user allocated with high power is decoded first and then the other user's data is decoded in 2 user scenario. It is observed that the user allocated with high power has the lower BER than the user with less power. An optisystem simulation results are discussed for both the user having different power.

\section{Declaration}

Conflict of interest All the authors declare that none of us have no conflict of interests.

Code availability Not applicable

\section{References}

Al-Ahmadi S, Maraqa O, Uysal M, et al (2018) Multi-user visible light communications: State-of-the-art and future directions. IEEE Access 6:70,55570,571

Barrami F, Le Guennec Y, Novakov E, et al (2014) An optical power efficient asymmetrically companded dco-ofdm for im/dd systems. In: 2014 23rd Wireless and Optical Communication Conference (WOCC), IEEE, pp 1-6 
Benjebbour A, Saito Y, Kishiyama Y, et al (2013) Concept and practical considerations of non-orthogonal multiple access (noma) for future radio access. In: 2013 International Symposium on Intelligent Signal Processing and Communication Systems, IEEE, pp 770-774

Chen C, Zhong WD, Yang H, et al (2017) On the performance of MIMONOMA-based visible light communication systems. IEEE Photonics Technology Letters 30(4):307-310

Deepthi S, Visalakshi P (2021) Enhanced optical ofdm: A novel approach for SISO and MIMO visible light communication system in indoor environment. Optical and Quantum Electronics 53(9):1-24

Dissanayake SD, Armstrong J (2013) Comparison of ACO-OFDM, DCOOFDM and ADO-OFDM in IM/DD systems. Journal of lightwave technology $31(7): 1063-1072$

Fu Y, Hong Y, Chen LK, et al (2018) Enhanced power allocation for sum rate maximization in OFDM-NOMA VLC systems. IEEE Photonics Technology Letters 30(13):1218-1221

Ghassemlooy Z, Popoola W, Rajbhandari S (2019) Optical wireless communications: system and channel modelling with Matlabß. CRC press

Hameed SM, Abdulsatar SM, Sabri AA (2021) Performance enhancement for visible light communication based ADO-OFDM

Jiang R, Sun C, Tang X, et al (2020) Joint user-subcarrier pairing and power allocation for uplink ACO-OFDM-NOMA underwater visible light communication systems. Journal of Lightwave Technology 39(7):1997-2007

Komine T, Nakagawa M (2004) Fundamental analysis for visible-light communication system using LED lights. IEEE transactions on Consumer Electronics 50(1):100-107

Lee D, Choi K, Kim KD, et al (2012) Visible light wireless communications based on predistorted OFDM. Optics Communications 285(7):1767-1770

Lian J, Brandt-Pearce M (2019) Multiuser visible light communications using OFDMA. In: 45th European Conference on Optical Communication (ECOC 2019), IET, pp 1-4

Lian J, Brandt-Pearce M (2020) Multiuser visible light communication systems using OFDMA. Journal of Lightwave Technology 38(21):6015-6023

Lin B, Ye W, Tang X, et al (2017) Experimental demonstration of bidirectional NOMA-OFDMA visible light communications. Optics express 25(4):43484355 
Liu X, Wang Y, Na Z (2019) Cooperative NOMA-based dco-ofdm vlc system. In: International Conference on Green Communications and Networking, Springer, pp 14-24

Marshoud H, Kapinas VM, Karagiannidis GK, et al (2015) Non-orthogonal multiple access for visible light communications. IEEE photonics technology letters 28(1):51-54

Marshoud H, Sofotasios PC, Muhaidat S, et al (2017) On the performance of visible light communication systems with non-orthogonal multiple access. IEEE Transactions on Wireless Communications 16(10):6350-6364

Mohsan SAH, Amjad H (2021) A comprehensive survey on hybrid wireless networks: practical considerations, challenges, applications and research directions. Optical and Quantum Electronics 53(9):1-56

Ren H, Wang Z, Han S, et al (2018) Performance improvement of M-QAM OFDM-NOMA visible light communication systems. In: 2018 IEEE Global Communications Conference (GLOBECOM), IEEE, pp 1-6

Rodoplu V, Hocaoğlu K, Adar A, et al (2020) Characterization of line-of-sight link availability in indoor visible light communication networks based on the behavior of human users. IEEE Access 8:39,336-39,348

Saju SC, George AJ, Koovapally-Kerala KK (2015) Comparison of ACOOFDM and DCO-OFDM in IM/DD systems. International Journal of Engineering Research \& Technology (IJERT) 4(04):1315-1318

Selvendran S, Raja AS, Muthu KE, et al (2019) Certain investigation on visible light communication with OFDM modulated white LED using optisystem simulation. Wireless Personal Communications 109(2):1377-1394

Shi J, Hong Y, Deng R, et al (2019) Demonstration of real-time software reconfigurable dynamic power-and-subcarrier allocation scheme for OFDMNOMA-based multi-user visible light communications. Journal of Lightwave Technology 37(17):4401-4409

Verma C, Selwal C (2018) 4QAM OFDM visible light communication using laser. In: 2018 7th International Conference on Reliability, Infocom Technologies and Optimization (Trends and Future Directions)(ICRITO), IEEE, pp 618-622

Wang JY, Wang JB, Wang Y (2017) Fundamental analysis for visible light communication with input-dependent noise. Optical Fiber and Wireless Communications pp 143-157 
Ye W, Chen J, Lin B, et al (2017) Experimental demonstration of NOMA visible light communications based on SCFDM. In: 2017 16th International Conference on Optical Communications and Networks (ICOCN), IEEE, pp $1-3$

Yeh CH, Chen HY, Chow CW, et al (2015) Utilization of multi-band OFDM modulation to increase traffic rate of phosphor-led wireless vlc. Optics express 23(2):1133-1138 